\title{
EDITOR'S MESSAGE / MESSAGE DE LA RÉDACTION
}

As I write my first editor's message, I would be remiss if I did not take this opportunity to thank Cari Merkley, previous Editor-in-Chief, for her hard work and dedication to this journal over the last 3 years. Cari moved this journal forward in a number of ways and continues to be a great source of support and advice. I would also like to take this opportunity to thank the entire editorial board who have worked very hard to get this issue published. This is our third issue and the culmination of the first full volume to be published under our entirely volunteer model. I am looking forward to an exciting and informative year.

The Canadian Health Libraries Association/ Association des bibliothèques de la santé du Canada (CHLA/ABSC) presented a number of awards this year and I would like to recognize those winners. This year's Hospital Librarian of the Year went to Angela Osterreicher from the University of Manitoba; the Emerging Leader Award went to Janice Kung from the University of Alberta; the David Crawford Honourary Life Membership Award went to Miriam Ticoll, a retired member; and the Margaret R. Charlton Award of Outstanding Achievement went to Dean Giustini from the University of British Columbia. The winners of the Student Paper Prize were Lara Maestro and Daniel Chadwick. Congratulations to all of our winners.

We have a great issue for you this time; not only does it include the student prize winning article on libraries' responses to the Truth and Reconciliation Report, we also have an article from the Oral Health Interest Group on academic dental librarianship. In addition, look for our book reviews and product reviews as well as a column on CHLA/ABSC's response to the Truth and Reconciliation Report.

\author{
Alison Farrell \\ JCHLA/JABSC Editor-in-Chief \\ Email:editor@chla-absc.ca
}

$\mathrm{Au}$ moment de rédiger mon premier message de la rédaction, il serait négligent de ma part de ne pas saisir l'occasion de remercier Cari Merkley, la rédactrice en chef qui m'a précédée, pour l'engagement et les efforts incessants dont elle a fait preuve au cours des trois dernières années. Cari a contribué à l'avancement du journal de diverses manières, et demeure toujours une source inestimable de soutien et de conseils. Je tiens aussi à profiter de l'occasion pour remercier tous les membres de l'équipe de rédaction qui ont travaillé d'arrache-pied pour réaliser la publication du présent numéro. C'est notre troisième numéro qui constitue l'apogée de notre tout premier volume à être entièrement publié selon le modèle de bénévolat. L'année qui vient s'annonce des plus emballantes et informatives.

L'Association des bibliothèques de la santé du Canada / The Canadian Health Libraries Association (ABSC / CHLA) a décerné plusieurs prix cette année et il convient de reconnaître ces lauréats. Le prix " Bibliothécaire de l'année en milieu hospitalier »a été décerné à Angela Osterreicher de l'Université du Manitoba ; le prix du « Flambeau de la relève » a été décerné à Janice Kung de l'Université de l'Alberta ; le prix « David Crawford - Membre honoraire à vie » a été décerné à Miriam Ticoll, membre à la retraite ; et le prix « Margaret Ridley Charlton pour réalisation exceptionnelle " a été décerné à Dean Giustini de l'Université de la Colombie Britannique. Les lauréats du prix «Exposé étudiant de l'ABSC / CHLA » sont Lara Maestro et Daniel Chadwick. Félicitations à tous nos gagnants!

Ce numéro se veut d'intérêt particulier. Non seulement y avons-nous incorporé l'article méritoire du prix Exposé étudiant de l'ABSC / CHLA portant sur les réactions des bibliothèques au rapport de la « Commission-vérité et réconciliation ", mais nous y avons aussi inclus un article en provenance du groupe d'intérêt sur la santé buccale traitant de la bibliothéconomie liée à la formation universitaire en dentisterie. De plus, jetez un œil sur nos critiques de livres et nos évaluations de produits, ainsi que sur une chronique portant sur la réponse de l'ABSC / CHLA au rapport de la « Commission-vérité et réconciliation ».

\author{
Alison Farrell \\ Rédactrice en chef, JABSC / JCHLA \\ Courriel: editor@chla-absc.ca
}

